\title{
Oscillating large-scale circulation in turbulent Rayleigh-Bénard convection
}

\author{
Jos Verdoold, ${ }^{*}$ Mark J. Tummers, and Kemo Hanjalić \\ Department of Multi-Scale Physics, Delft University of Technology, Lorentzweg 1, 2628 CJ Delft, The Netherlands
}

(Received 27 January 2006; published 10 May 2006)

\begin{abstract}
We report on the dynamics and structure of the turbulent velocity field in a high-Rayleigh-number $\left(\mathrm{Ra}=5.9 \times 10^{8}\right)$ thermal convection cell with an aspect ratio of 4 . Spectral density functions (measured with laser Doppler velocimetry) indicated the existence of a large-scale periodic component. The long-time mean flow field (measured with particle image velocimetry) revealed that the large-scale circulation in the aspectratio-4 cell consists of two corotating rolls. The periodicity in the flow could be traced back to the alternating growth and decay of these rolls.
\end{abstract}

DOI: 10.1103/PhysRevE.73.056304

PACS number(s): 47.20.Bp, 44.25.+f, 47.27.te

Rayleigh-Bénard convection (RBC) in a fluid layer between two horizontal surfaces heated from below and cooled from above has long been regarded as a paradigm of many examples of thermal convection encountered in nature and in manmade environments. $\mathrm{RBC}$ is characterized by the Rayleigh number $\mathrm{Ra}=g \alpha \Delta T H^{3} /(\kappa \nu)$, the Prandtl number $\operatorname{Pr}=\nu / \kappa$, and the aspect ratio $\Gamma=W / H$. Here, $g$ is the gravitational acceleration, $\Delta T$ is the temperature difference between the plates, $H$ is the distance between the plates, and $W$ is the lateral extent of the plates. The symbols $\alpha, \nu$, and $\kappa$ are the fluid's thermal expansion coefficient, kinematic viscosity, and thermal diffusivity, respectively. Despite the simplicity of the geometry and the boundary conditions and extensive studies, many physical phenomena in RBC are not fully understood [1,2]. Among the most debatable issues is the scaling law for heat transfer, especially above approximately $\mathrm{Ra}=4 \times 10^{7}$ (see Niemela et al. [3]). Underlying the discussion about the appropriate value of the exponent of the $\mathrm{Ra}$ number are the possible differences in the dynamics of the turbulence and large-scale convective structures, which may be easily overlooked in experimental studies of the global heat transfer without having sufficient insight into the flow and turbulence field. Experiments in RBC cells have been conducted in a wide variety of $\mathrm{Ra}$, aspect ratio, and flow media, but detailed quantitative studies of the turbulent flow field are scarce, and, as pointed out by Qiu and Tong [4], often hampered by the use of suboptimal measurement techniques. In a transparent fluid like water, the techniques of choice for velocity measurements are laser Doppler velocimetry (LDV) and particle image velocimetry (PIV). Both techniques are nonintrusive. The strength of the latter lies in its ability to capture (instantaneous) velocity fields, while the former is a single-point technique capable of measuring velocity time series with superior spatial and temporal resolution. LDV has been applied to study RBC by Qiu et al. $[4,5]$ and Shang et al. [6], whereas PIV has been conducted by Prasad and Gonuguntla [7], Xia et al. [8], and Sun et al. [9].

In this paper we report on experimentally detecting an oscillating convective pattern from long-term velocity measurements in an aspect-ratio-4 $(\Gamma=4) \mathrm{RBC}$ cell at

\footnotetext{
*Electronic address: j.verdoold@ws.tn.tudelft.nl
}

$\mathrm{Ra}=5.9 \times 10^{8}$. Grossmann and Lohse [10] argue that the majority of studies on RBC concentrate on cells with an aspect ratio of approximately 1 , and that future experiments on RBC should be aimed at large aspect ratios. The present experiment can be seen as a step in that direction. Experiments with rectangular RBC cells of larger aspect ratios have been reported before, e.g., Fitzjarrald [11] and Ciliberto et al. [12] report on (traversing) point measurements. However, to the author's knowledge, there is no detailed information in the literature on the turbulent flow field in RBC cells with $\Gamma=4$ at similar Rayleigh and Prandtl numbers. The focus of the present study is on the large-scale convection (LSC), also known as "wind," and the origin of the low-frequency oscillation in this large-scale flow. It will be shown that the LSC in the $\Gamma=4$ cell shares certain characteristics with the $\Gamma=1$ cell, but exciting differences occur as well.

The $\mathrm{RBC}$ results reported here have been obtained in a $600 \times 600 \times 155 \mathrm{~mm}^{3}(W \times W \times H)$ cell filled with water. Two copper plates at the top and at the bottom are kept at a constant temperature by passing water through them from two basins containing constant temperature water. By doing so, the plates impose a controlled temperature difference on the working fluid. Experiments were conducted at a Rayleigh number $\mathrm{Ra}=5.9 \times 10^{8}$, corresponding to a temperature difference of $6.3{ }^{\circ} \mathrm{C}$. The mean temperature of the bulk fluid was approximately $30{ }^{\circ} \mathrm{C}$ corresponding to a Prandtl number $\operatorname{Pr}=\nu / \kappa=5.5$. Throughout this paper we will use a Cartesian coordinate system with its origin at the center of the bottom wall. The $x$ and $y$ coordinates are measured along the bottom wall, parallel to the side walls of the RBC cell. The $z$ coordinate measures the wall-normal distance.

Single-point measurements of the velocity were carried out by using a two-component LDV system manufactured by Dantec. The green $(514.5 \mathrm{~nm})$ and blue $(488.0 \mathrm{~nm})$ colors of a $5 \mathrm{~W}$ argon-ion laser were used to measure two velocity components simultaneously. One beam of each color was frequency preshifted by a Bragg cell to enable the detection of instantaneous flow reversals. The two beam pairs were oriented such that two wall-parallel velocity components could be measured directly. Sometimes the optical configuration was changed such that the wall-normal and a wallparallel velocity component were measured directly. The length and diameter of the two measurement volumes are 0.8 


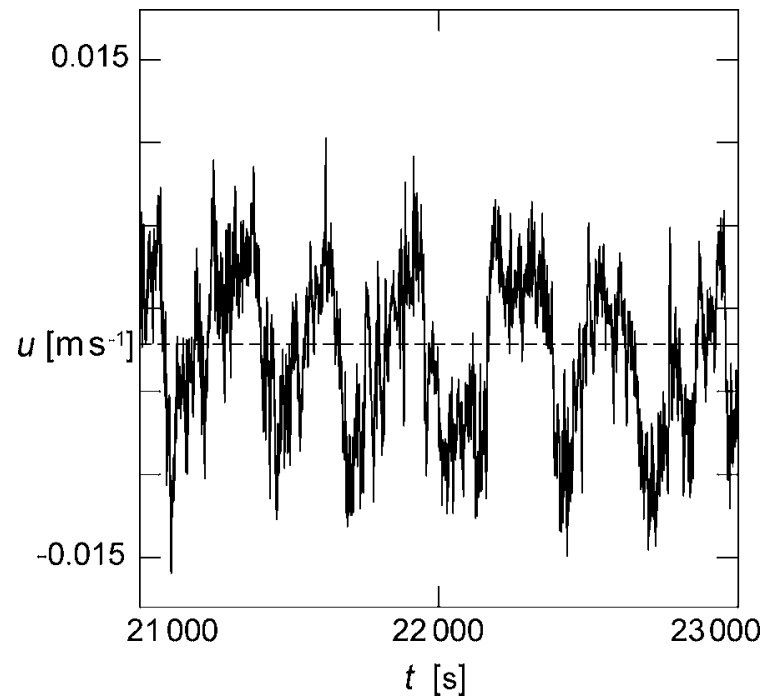

FIG. 1. Wall-parallel velocity component $u$ as a function of time. Dashed line indicates the average velocity.

and $0.1 \mathrm{~mm}$, respectively, which is sufficiently small to resolve the smallest length scales of the flow. The light scattered by $5 \mu \mathrm{m}$ polyamide seed particles (Dantec) was collected in the forward direction. The photomultiplier output signals were fed to two Dantec BSA signal processors to determine the instantaneous velocities. The LDV system measured the instantaneous velocities of the seed particles with an error of $0.5 \%$.

Velocity fields were measured by using a PIV system that included a Continuum Minilite double-pulsed neodymiumdoped (Nd:YAG) laser with a pulse energy of $25 \mathrm{~mJ}$. This laser was used to make an approximately 1 -mm-thick light sheet that illuminated the seeding particles in the flow. Hollow glass spheres (Potters-Ballotini) with a diameter of approximately $10 \mu \mathrm{m}$ were used as seed particles. Two PCO Sensicam cameras with a resolution of $1280 \times 1024$ pixels recorded images of the seed particles in the light sheet. A 28 -mm-focal-length lens with a numerical aperture $f=4$ was mounted on each camera. The time separation between the two consecutive images was set to $25 \mathrm{~ms}$. The image pairs were analyzed in three subsequent steps using Gaussian local median filtering and interpolation for interrogation areas of $16 \times 16$ pixels. The resulting vector field covered the complete RBC cell with a resolution of $0.5 \times 0.5 \mathrm{~cm}^{2}$.

LDV measurements were taken at various points in the cell, but for brevity we present results only for one point at $x / W=0.17, y=0, z / H=0.22$. At this point approximately $2.8 \times 10^{6}$ samples were taken at a mean rate of $16 \mathrm{~Hz}$, resulting in a long measuring time of approximately $49 \mathrm{~h}$. The average of the velocity signal is $-2.15 \mathrm{~mm} \mathrm{~s}^{-1}$. A 2000 -slong segment of the velocity time series for the wall-parallel velocity component is shown in Fig. 1. The graph indicates the presence of a large-scale quasiperiodic motion. This was investigated further by computing the spectral density function (SDF) for the entire time series, which is defined as the Fourier transform of the autocorrelation function $\rho(\tau)$ $=\overline{u^{\prime}(t) u^{\prime}(t+\tau)} / \overline{u^{\prime 2}}$, where $u^{\prime}(t)[\equiv u(t)-\bar{u}]$ is a fluctuating component of the turbulent velocity, $t$ is time, $\tau$ is the time

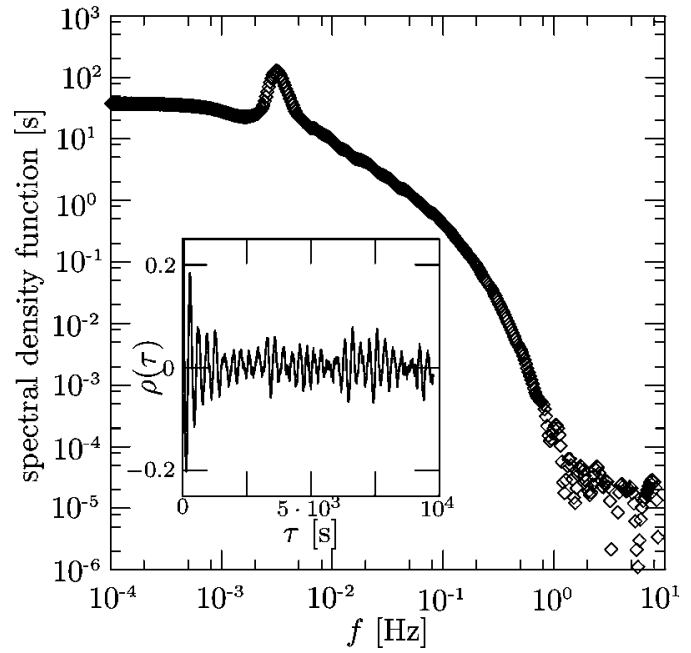

FIG. 2. Spectral density function of the velocity component in the $x$ direction measured at $x / W=0.17, y=0, z / H=0.22$. Inset: autocorrelation function for the same position.

lag, and the overbar denotes ensemble averaging. Computing the SDF from LDV velocity samples requires a special algorithm that takes into account the randomness of the sampling times and the velocity bias. In the present work the SDF was determined from the algorithm described by Tummers and Passchier [13]. The resulting SDF, shown in Fig. 2, indicates that the large-scale motion has a periodicity of approximately 308 s. The inset in Fig. 2 shows that the oscillations in the $\mathrm{ACF}$ are maintained for a very long time with the amplitude varying irregularly as reported by Funfschilling and Ahlers [14]. From integration of the different parts of the measured SDF one can determine the contribution of the periodicity to the total power of the velocity fluctuations. It is found that approximately $20 \%$ of the total power is due to the periodicity, which is consistent with the amplitude of the ACF.

The periodic motion is a well-known phenomenon in $\mathrm{RBC}$ for $\mathrm{Ra}>4 \times 10^{7}$. It has been reported in literature (see, for example, Castaing et al. [15] or Qiu et al. [5]) that the periodicity in RBC cells with an aspect ratio of about 1 is related to the formation of a LSC. The interaction between this LSC and the thermal plume formation is a topic of intense research, and, to the author's knowledge, this is the first time that a similar periodicity has been reported in a RBC cell with an aspect ratio 4.

Inspired by the above observations the following questions arose. Is there a LSC in the aspect-ratio-4 cell? If so, what form does it have? Furthermore, is there a relation between the LSC and the periodicity, and is this relation different from that in an aspect-ratio-1 cell? To answer these questions we focused on the turbulent flow structures. Since it is difficult to study these flow structures from a single-point measurement technique (such as LDV), it was decided to deploy PIV. Our PIV system was used to collect a series of $3.0 \times 10^{4}$ snapshots of the velocity field in the $x, z$ plane at a rate of $1 \mathrm{~Hz}$. The long-time mean flow field is shown in Fig. 3. Inspection of this mean flow field has resulted in the qualitative sketch of the streamline pattern that is shown in Fig. 4. There is a flow along the periphery of the cell that encloses 


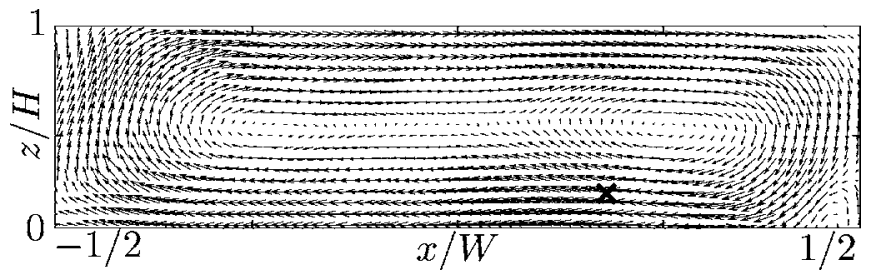

FIG. 3. Mean flow field in the $x, z$-plane. The cross at $x / W$ $=0.17, z / H=0.22$ indicates the LDV position.

two corotating rolls (centers) and a saddle point.

The LSC is "weak" in the sense that the long-time mean velocity is small compared to the magnitude of the instantaneous velocity fluctuations, as can be readily observed from Fig. 1. Note that this is in contrast to the situation in an aspect-ratio-1 cell where the LSC can be so strong that the instantaneous velocity only seldom changes sign [6]. As a consequence of the weak circulation, the mean flow field shown in Fig. 3 cannot be observed from the instantaneous PIV snapshots. It emerges only after averaging over a very long time.

For each PIV interrogation area one can extract a velocity time series from the PIV measurements. Clearly, the velocity samples in these time series are taken at regular intervals of $1 \mathrm{~s}$, and standard data processing methods can be applied to compute the SDF. It was checked that the peak in the SDFs determined from the PIV time series was the same as that determined from the LDV data. Moreover, the SDFs determined from the PIV time series extracted at various locations in the RBC cell showed conclusively that the period of the oscillation was the same everywhere, thus proving that the oscillation is a global phenomenon.

From flow and temperature visualizations using thermochromic liquid crystal tracers, we have observed that groups of plumes tend to move along the side walls of the RBC cell, not dissimilar to what has been reported by Qui et al. [5] for aspect-ratio- 1 cells. They suggested that the buoyancy forces exerted on groups of plumes that rise or fall along the sidewalls is what drives the LSC. We find the same mechanism at work in the present $\mathrm{RBC}$ cell, but there are also interesting differences. As mentioned before, the LSC is weak in the present $\mathrm{RBC}$ cell, and plumes that are released from the horizontal walls are not strongly sheared. As a consequence, not all the plumes are carried toward the sidewalls before they move up (or down). The weak circulation allows the plumes to be released from the thermal boundary layers on the horizontal walls and move up (or down) long before reaching the sidewalls. We did not observe individual plumes reaching the opposite wall by traversing the turbulent core, but groups of plumes regularly form temporary pathways between the horizontal plates. The location, size, and orientation of these

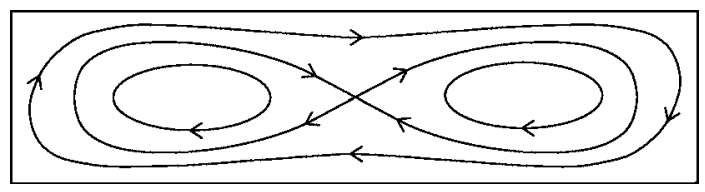

FIG. 4. Sketch of the mean flow field in the $\Gamma=4$ cell.
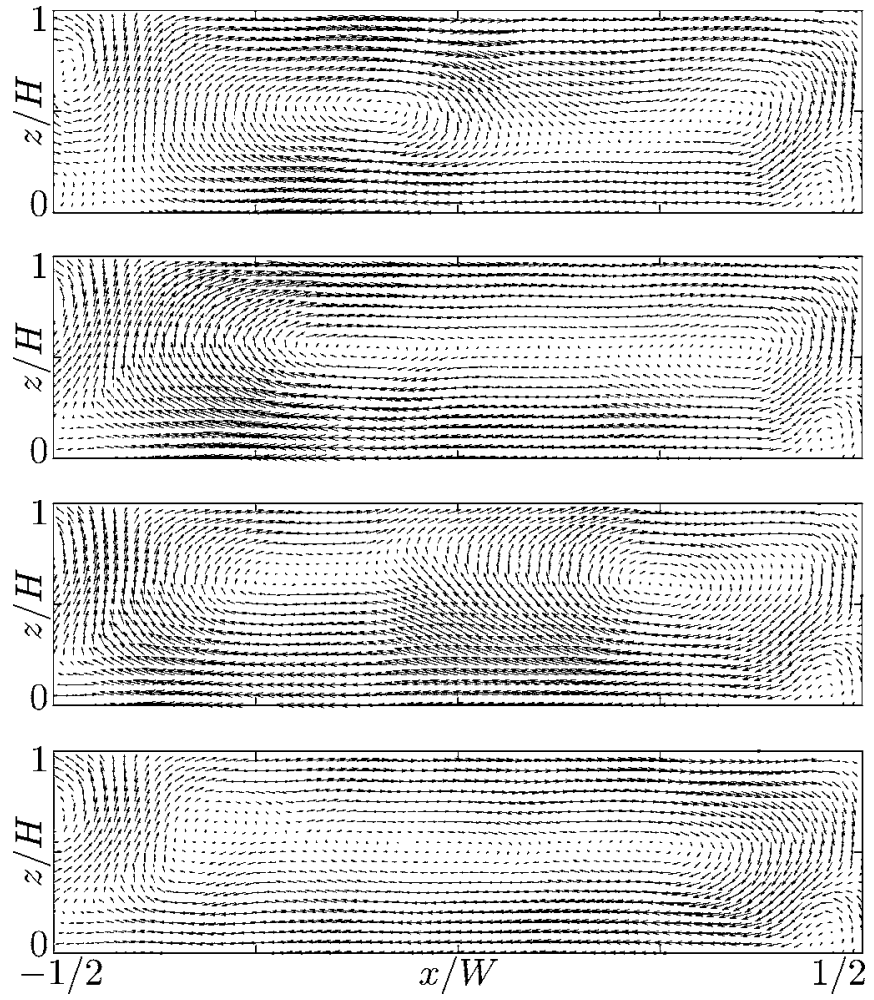

FIG. 5. Phase-resolved velocity fields for $N=4$.

pathways are not steady. Their lifetimes are of the same order of magnitude as the period of the oscillations, but generally shorter. It is speculated here that the two corotating rolls in the long-time mean flow field in Fig. 3 occur due to the formation of these pathways at preferential locations. In other words, one cannot predict when and where a pathway is formed, nor can one predict its size, strength and duration, but the long-time mean flow indicates that the pathways have a preferred location near the centre of the cell.

For the aspect-ratio-1 cell, Villermaux [16] proposed that the interaction between the thermal boundary layers on the upper and lower walls depends on the delay time $H / U$ where $U$ is the large-scale velocity. Qui et al. [17] modified this view slightly by pointing out that $U$ is actually a fluctuating quantity, but in essence, the oscillations observed in the $\Gamma=1$ cell are proportional to $U / H$, so that a physical picture arises with alternating eruptions of groups of plumes from the thermal boundary layers. These groups move up or down in opposing side wall regions thus driving a LSC that takes the form of a single roll.

To determine a relation between the LSC, the plume motion and the low-frequency oscillation for the present aspectratio- 4 cell, we decided to resolve the instantaneous velocity fields (i.e., the PIV snapshots) into phases. Phase-resolved velocity fields were determined as follows. The series of $3 \times 10^{4}$ instantaneous velocity fields was split into segments of 308 sequential snapshots thus matching the measured period. Each segment was subsequently divided into $N$ intervals of equal length. The phase-resolved mean velocity field for the $i$ th phase followed from the mean of all snapshots in the $i$ th interval in each of the segments. Figure 5 shows the phase-resolved velocity fields for $N=4$. In phase 1 (top inset) 
one can observe the saddle point and the two rolls with the left roll being much stronger than the right roll. The flow is along the circumference of the RBC cell, but there is also a tendency for the fluid to cross the cell along a "descending diagonal" located on the right-hand side of the strongest roll. During phase 2 the fluid no longer descends along this diagonal, but most of the momentum is still concentrated on the left-hand side of the RBC cell in the remnant of the left roll. A strengthening of the right roll sets in, and, during phase 3 fluid moves along the circumference and it can cross the RBC cell along an "ascending diagonal" on the left-hand side of the right roll. During phase 4 the right roll loses its strength, and the process repeats itself. The driving mechanism for the LSC are the buoyancy forces exerted on groups of plumes that move up (or down) in regions that are separated laterally, effectively providing a torque. These groups of ascending (or descending) plumes occur at two locations: (1) near the sidewalls as in the aspect-ratio-1 cell, and (2) at locations away from the sidewalls. The latter provides an additional channel for the transport of heat between the two horizontal plates that does not occur in the aspect-ratio-1 cell. Although the exact location of these channels cannot be predicted, they appear to occur most frequently around the half-width of the cell, thus forming "short" rolls with lateral
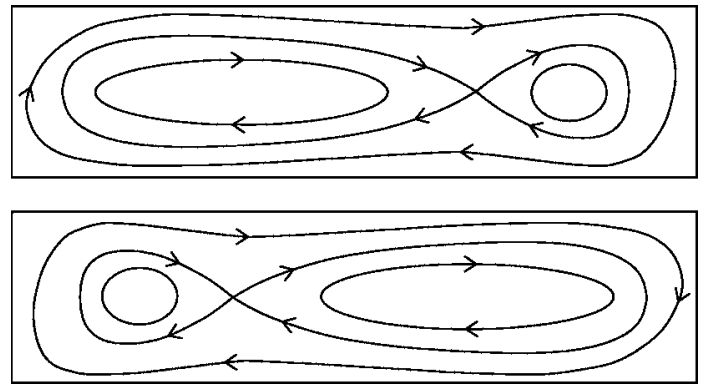

FIG. 6. Sketch of two extreme states of the LSC with half a period phase difference.

size of about $2.5 \mathrm{H}$ that alternatingly appear on the left or on the right of the cell.

In summary, the experiments reveal that the LSC in the aspect-ratio-4 RBC cell consists of a flow around the periphery that encloses two corotating rolls. Using a phase resolving technique, it is shown that the growth and decay of the rolls produce a well-defined period that is maintained for a very long time. Figure 6 sketches two extremes in the temporal evolution of the LSC that have a phase difference of half a period, thus illustrating the nature of the oscillation in the aspect-ratio-4 cell.
[1] E. D. Siggia, Annu. Rev. Fluid Mech. 26, 137 (1994).

[2] L. P. Kadanoff, Phys. Today 54(8), 34 (2001).

[3] J. J. Niemela, L. Skrbek, K. R. Sreenivasan, and R. Donnelly, Nature (London) 404, 837 (2000).

[4] X.-L. Qiu and P. Tong, Phys. Rev. E 64, 036304 (2001).

[5] X.-L. Qiu, S. H. Yao, and P. Tong, Phys. Rev. E 61, R6075 (2000).

[6] X.-D. Shang, X.-L. Qiu, P. Tong, and K.-Q. Xia, Phys. Rev. Lett. 90, 074501 (2003).

[7] A. K. Prasad and P. V. Gonuguntla, Phys. Fluids 8, 2460 (1996).

[8] K.-Q. Xia, C. Sun, and S.-Q. Zhou, Phys. Rev. E 68, 066303 (2003)

[9] C. Sun, K.-Q. Xia, and P. Tong, Phys. Rev. E 72, 026302 (2005)
[10] S. Grossmann and D. Lohse, J. Fluid Mech. 486, 105 (2003).

[11] D. E. Fitzjarrald, J. Fluid Mech. 73, 693 (1976).

[12] S. Ciliberto, S. Cioni, and C. Laroche, Phys. Rev. E 54, R5901 (1996).

[13] M. J. Tummers and D. M. Passchier, Meas. Sci. Technol. 12, 1641 (2001).

[14] D. Funfschilling and G. Ahlers, Phys. Rev. Lett. 92, 194502 (2004).

[15] B. Castaing, G. Gunaratne, F. Heslot, L. Kadanoff, A. Libchaber, S. Thomae, X.-Z. Wu, S. Zaleski, and G. Zanetti, J. Fluid Mech. 204, 1 (1989).

[16] E. Villermaux, Phys. Rev. Lett. 75, 4618 (1995).

[17] X.-L. Qiu, X. D. Shang, P. Tong, and K.-Q. Xia, Phys. Fluids 16, 412 (2004). 